\title{
Fabrication of Large Area Printable Composite Magnets
}

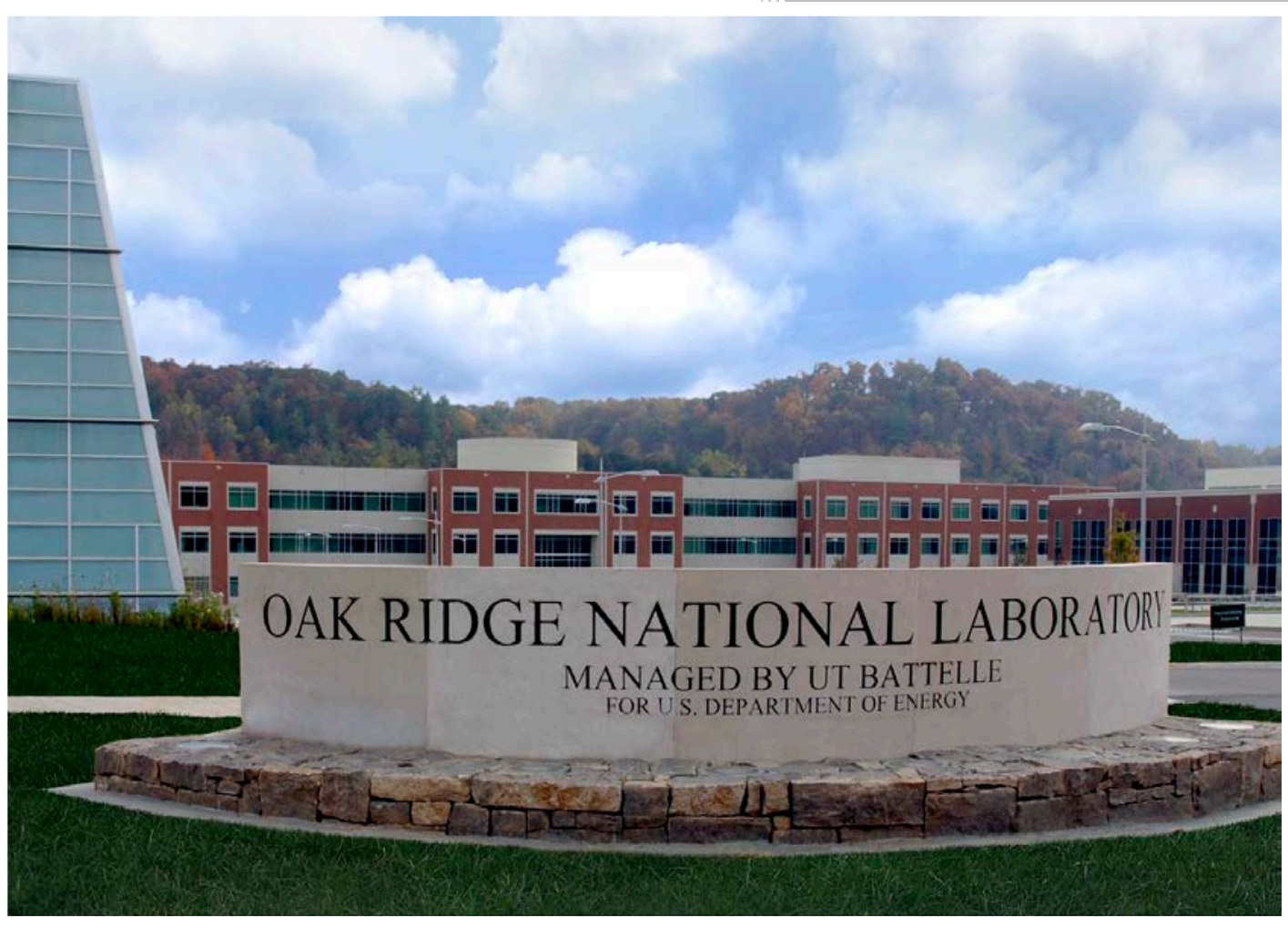

M. Parans Paranthaman

$09 / 29 / 2016$

CRADA FINAL REPORT

NFE-15-05779

Approved for Public Release. Distribution is Unlimited. 


\section{DOCUMENT AVAILABILITY}

Reports produced after January 1, 1996, are generally available free via US Department of Energy (DOE) SciTech Connect.

Website http://www.osti.gov/scitech/

Reports produced before January 1, 1996, may be purchased by members of the public from the following source:

National Technical Information Service

5285 Port Royal Road

Springfield, VA 22161

Telephone 703-605-6000 (1-800-553-6847)

TDD 703-487-4639

Fax 703-605-6900

E-mail info@ntis.gov

Website http://www.ntis.gov/help/ordermethods.aspx

Reports are available to DOE employees, DOE contractors, Energy Technology Data Exchange representatives, and International Nuclear Information System representatives from the following source:

Office of Scientific and Technical Information

PO Box 62

Oak Ridge, TN 37831

Telephone 865-576-8401

Fax 865-576-5728

E-mail reports@osti.gov

Website http://www.osti.gov/contact.html

This report was prepared as an account of work sponsored by an agency of the United States Government. Neither the United States Government nor any agency thereof, nor any of their employees, makes any warranty, express or implied, or assumes any legal liability or responsibility for the accuracy, completeness, or usefulness of any information, apparatus, product, or process disclosed, or represents that its use would not infringe privately owned rights. Reference herein to any specific commercial product, process, or service by trade name, trademark, manufacturer, or otherwise, does not necessarily constitute or imply its endorsement, recommendation, or favoring by the United States Government or any agency thereof. The views and opinions of authors expressed herein do not necessarily state or reflect those of the United States Government or any agency thereof. 
Chemical Sciences Division

Advanced Manufacturing Office

\title{
Fabrication of Large Area Printable Composite Magnets
}

M. Parans Paranthaman ${ }^{1}$, Ling Li ${ }^{1}$, Orlando Rios ${ }^{1}$, Chad Duty ${ }^{1}$, Brian Post ${ }^{1}$, Vlastimil Kunc ${ }^{1}$, Robert Springfield ${ }^{2}$, Josh Martin ${ }^{2}$, and Rick Spears ${ }^{2}$

${ }^{1}$ Oak Ridge National Laboratory, Oak Ridge, TN 37831, USA

${ }^{2}$ Tru-Design LLC, Knoxville, TN 37983, USA

Date Published: 09/29/2016

\author{
Prepared by \\ OAK RIDGE NATIONAL LABORATORY \\ Oak Ridge, Tennessee 37831-6283 \\ managed by \\ UT-BATTELLE, LLC \\ for the \\ US DEPARTMENT OF ENERGY \\ under contract DE-AC05-00OR22725
}

This report does not contain Protected CRADA information

Approved for Public Release 


\section{CONTENTS}

\section{Page}

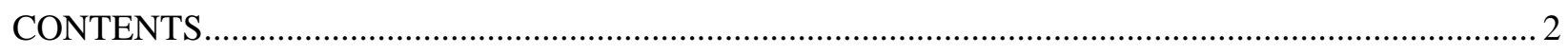

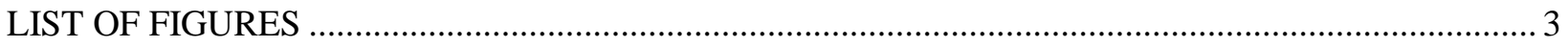

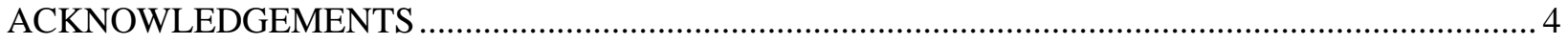

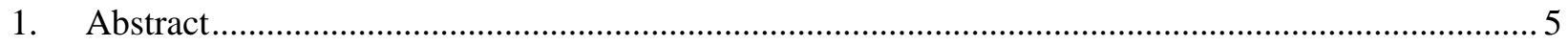

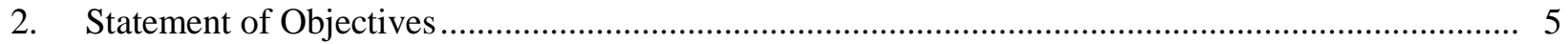

3. Benefits to the Funding DOE Office's Mission …......................................................................... 5

4. Technical Discussion of Work Performed by All Parties ................................................................ 6

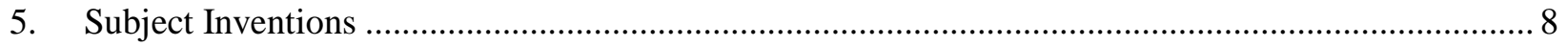

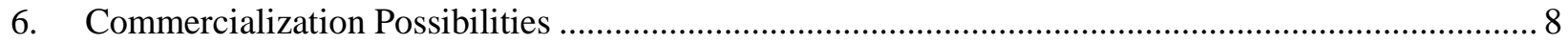

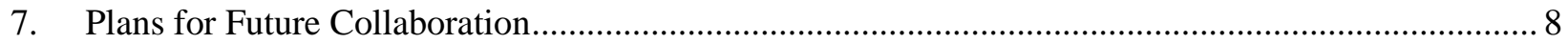

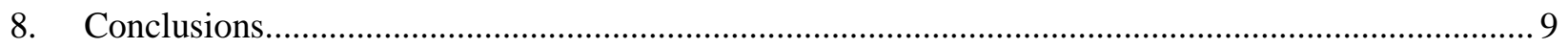

9. References........................................................................... 9 


\section{LIST OF FIGURES}

Fig. 1. Images of (a) as-printed 6” diameter x 6” tall cylinder of BAAM isotropic NdFeB nylon composite magnets; and (b) after slicing the cylinder into two magnetic braces.

Fig. 2. Magnetic properties of BAAM fabricated isotropic NdFeB nylon composite magnets (left) and room temperature maximum energy product (right) for the BAAM magnet. All the magnetic properties are tabulated in a table on the top.

Fig. 3. Images of 1.5” x 1.5” composite magnet fabricated with anisotropic MQA NdFeB magnet powders and a resin without any magnetic field (left) and with magnetic field (right).

Fig. 4. Magnetic hysteresis loops of the composite magnet fabricated with anisotropic MQA NdFeB magnet powders and a resin with and without any magnetic field. 


\section{ACKNOWLEDGEMENTS}

This CRADA NFE-15-05779 was conducted as a Technical Collaboration project within the Oak Ridge National Laboratory (ORNL) Manufacturing Demonstration Facility (MDF) sponsored by the US Department of Energy Advanced Manufacturing Office (CPS Agreement Number 24761). Opportunities for MDF technical collaborations are listed in the announcement "Manufacturing Demonstration Facility Technology Collaborations for US Manufacturers in Advanced Manufacturing and Materials Technologies” posted at http://web.ornl.gov/sci/manufacturing/docs/FBO-ORNL-MDF-2013-2.pdf. The goal of technical collaborations is to engage industry partners to participate in short-term, collaborative projects within the Manufacturing Demonstration Facility (MDF) to assess applicability and of new energy efficient manufacturing technologies. Research sponsored by the U.S. Department of Energy, Office of Energy Efficiency and Renewable Energy, Advanced Manufacturing Office, under contract DEAC05-00OR22725 with UT-Battelle, LLC. Thanks are due to Nadya Ally with slicing the BAAM magnets.

Notice: This manuscript has been authored by UT-Battelle, LLC under Contract No. DE-AC0500OR22725 with the U.S. Department of Energy. The United States Government retains and the publisher, by accepting the article for publication, acknowledges that the United States Government retains a non-exclusive, paid-up, irrevocable, world-wide license to publish or reproduce the published form of this manuscript, or allow others to do so, for United States Government purposes. The Department of Energy will provide public access to these results of federally sponsored research in accordance with the DOE Public Access Plan (http://energy.gov/downloads/doe-public-access-plan). 


\section{Abstract}

The technical objective of this technical collaboration phase I proposal was to fabricate large area NdFeB composite magnets at the Oak Ridge National Laboratory Manufacturing Demonstration Facility (ORNL MDF). The goal was to distribute domestically produced isotropic and highly anisotropic high energy density magnetic particles throughout the composite structure in order to enable site specific placement of magnetic phases and minimize the generated waste associated with permanent magnet manufacturing. Big area additive manufacturing (BAAM) and magnet composite fabrication methods were used in this study. BAAM was used to fabricate $65 \mathrm{vol} \%$ isotropic MQP NdFeB magnets in nylon polymer matrix. BAAM magnet cylinder was sliced to two magnetic arc-shaped braces. The density of the small BAAM magnet pieces reached $4.1 \mathrm{~g} / \mathrm{cm}^{3}$, and the room temperature magnetic properties are: Intrinsic coercivity $H_{c i}=8.8$ kOe, Remanence $B_{r}=4.2 \mathrm{kG}$, and energy product $(B H)_{\max }=3.7$ MGOe. Also, 1.5” x 1.5” composite magnets with anisotropic MQA NdFeB magnet in a resin were also fabricated under magnetic field. The unaligned sample had a density of $3.75 \mathrm{~g} / \mathrm{cm}^{3}$. However, aligned sample possessed a density of 4.27 $\mathrm{g} / \mathrm{cm}^{3}$. The magnetic properties didn't degrade during this process. This study provides a pathway for preparing composite magnets for various magnetic applications.

\section{Statement of Objectives}

This phase I technical collaboration project (MDF-TC-2016-63) was started on July 31, 2015 and was completed on September 30, 2016. The collaboration partner, Tru-Design, LLC is a small business and has established itself as the leader in fabricating carbon fiber composites that will aid in conserving energy and natural resources for the benefit of our economy. They apply innovation to develop creative solutions with composites. Their resources and abilities to create molds, place them in a position of demand by both manufacturers and developers. This project focuses on one of the magnet applications: the fabrication of an advanced magnetic brace system that allows patients with cerebral palsy (CP) to walk better. These braces rely upon the repulsive force of aligned magnetic materials to maintain separation of a patient's knees during normal walking, which is a common challenge for patients with CP. The brace would require the deposition of a permanent magnet that is capable of being shaped to match the complex surface of the human anatomy. The main objective of this project is to use additive manufacturing technologies to fabricate composite magnets. The magnetic properties of the fabricated composite magnets have been characterized and fully evaluated.

\section{Benefits to the Funding DOE Office's Mission}

Sintered $\mathrm{Nd}_{2} \mathrm{Fe}_{14} \mathrm{~B}(\mathrm{NdFeB})$ magnets are the strongest permanent magnets available today. These magnets are made by powder metallurgical techniques which include: (1) melting and casting the alloy with a composition close to $\mathrm{Nd}_{2} \mathrm{Fe}_{14} \mathrm{~B}$, (2) fine graining the product by hydrogen decrepitation, and milling (3) aligning and consolidating the powders by pressing in a magnetic field, (4) sintering at high temperature $\left(\sim 1100{ }^{\circ} \mathrm{C}\right)$ to obtain dense bodies, (5) annealing at a lower temperature $\left(\sim 600{ }^{\circ} \mathrm{C}\right)$ to refine magnetic properties, (6) finishing which involves cutting and/or grinding to shape and finally (7) coating. During step 6 a significant fraction, 30 to $50 \%$, of the material is reduced to heavily contaminated and oxidized grinding swarf which is very difficult to recycle. The high cost of rare earth raw materials and issues related to adequate and dependable supply of heavy rare earths, e.g. dysprosium, are holding back the adoption or expanded use of these technologies. However if advanced additive manufacturing techniques can be used to fabricate composite magnets based device structures with high yield using unique capabilities that exist within the Manufacturing Demonstration Facility (MDF) at ORNL. This project complements the method developed to fabricate bonded magnets based on anisotropic NdFeB magnet 
powders through the Critical Materials Institute (CMI) hub project. Overall, the advancement of the utilization of the additive techniques could lead to the adoption of additive manufacturing technology by magnet manufacturing companies, and lead to job growth and higher US global manufacturing competitiveness.

\section{Technical Discussion of Work Performed by All Parties}

The Big Area Additive Manufacturing (BAAM) system located at the MDF at ORNL was utilized to fabricate complex isotropic NdFeB magnets. The BAAM system deposits high-performance engineered thermoplastics and customized thermoplastic composites via a melt extrusion processing, which enables rapid manufacturing of parts completely unbounded in size. The feedstock materials used here are magnetic pellets composed of 65 vol\% isotropic MQP NdFeB powder and 35 vol\% Nylon- 12 . As shown in Fig. 1 (a), composite magnets with a cylindrical shape was fabricated with the BAAM. After printing, the cylinder was sliced to prepare a magnetic brace (Fig. 1 (b)).

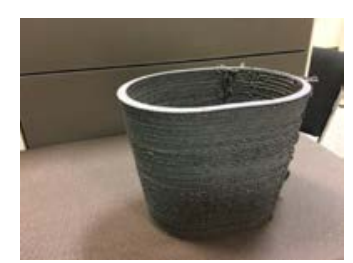

(a) As-printed BAAM magnet
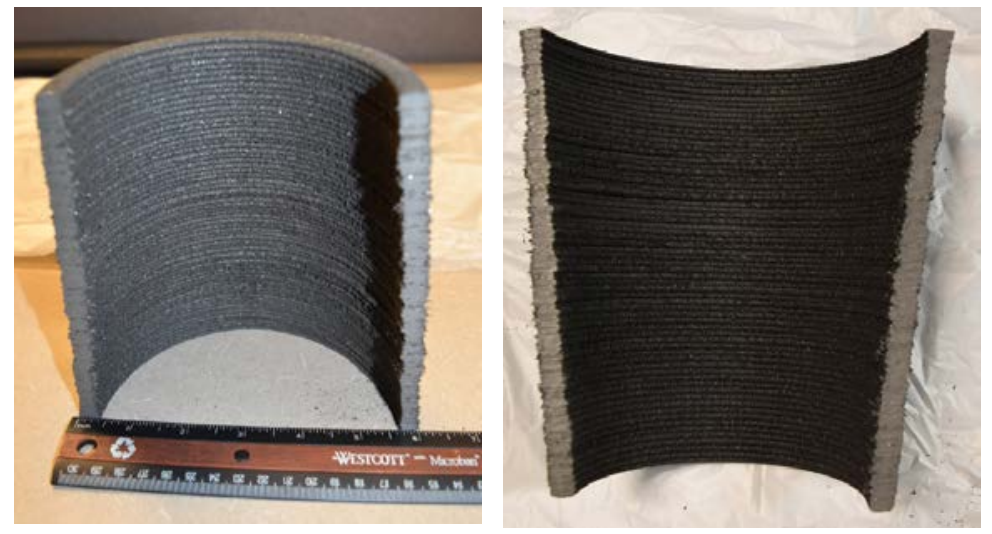

(b) After slicing the BAAM magnet

Fig. 1. Images of (a) as-printed $~ 6$ " diameter x 6" tall cylinder of BAAM isotropic NdFeB nylon composite magnets; and (b) after slicing the cylinder into two magnetic braces.

The magnetic properties of the BAAM fabricated magnets were investigated. The field dependence flux densities for the BAAM magnet are shown in Fig. 2. The magnetic properties of the BAAM magnet do not show any noticeable degradation compared to those of the starting pellets. The density of the BAAM magnet is $4.1 \mathrm{~g} / \mathrm{cm}^{3}$. The BAAM magnet has the intrinsic coercivity $H_{c i}=8.8 \mathrm{kOe}$, remanence $B_{r}=4.2$ $\mathrm{kG}$, and energy product $(B H)_{\max }=3.7$ MGOe. The clear coating developed to complete the surface finish for binderjet magnets can be used for BAAM magnets as well [1]. 


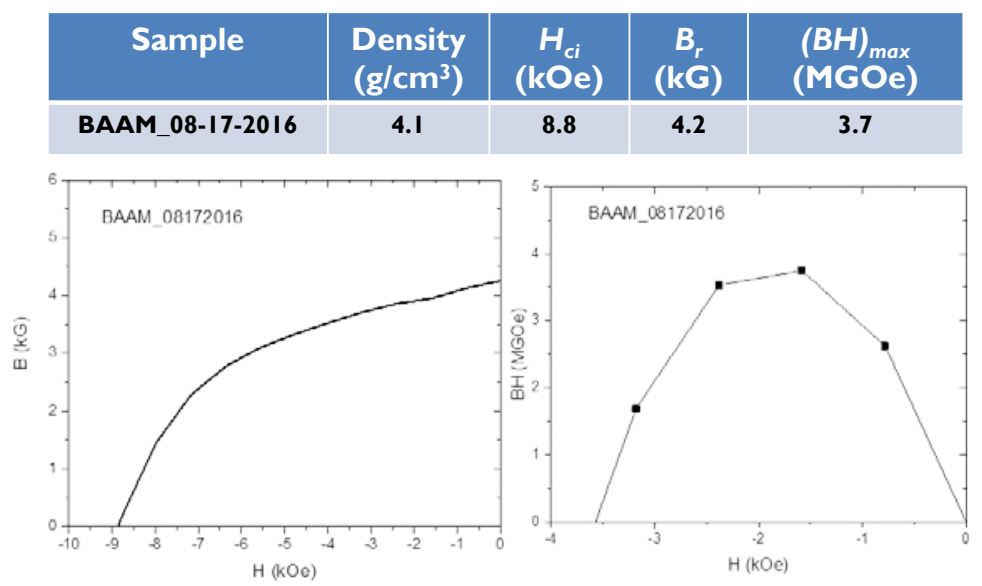

Fig. 2. Magnetic properties of BAAM fabricated isotropic NdFeB nylon composite magnets (left) and room temperature maximum energy product (right) for the BAAM magnet. All the magnetic properties are tabulated in a table on the top.

As shown in Fig. 3, 1.5” x 1.5” composite magnets with anisotropic MQA NdFeB magnets in a resin were also fabricated using a batch process. Carbon fiber liner is incorporated into the composite magnets to improve the mechanical properties. During the curing process, magnetic fields are used to align the anisotropic magnets. Further optimization is necessary to fully align the magnets and achieve high energy product. The unaligned sample had a density of $3.75 \mathrm{~g} / \mathrm{cm}^{3}$. However, aligned sample possessed a density of $4.27 \mathrm{~g} / \mathrm{cm}^{3}$. As shown in Fig. 4, the magnetic properties didn't degrade during this process. This study provides a pathway for preparing composite magnets for various magnetic applications.

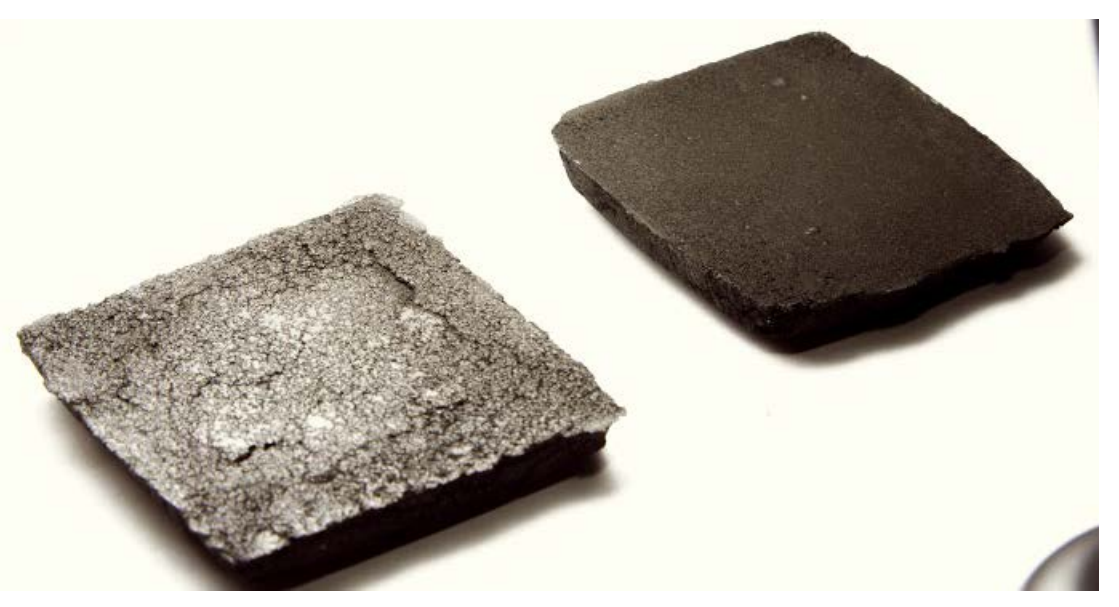

Fig. 3. Images of 1.5” x 1.5” composite magnet fabricated with anisotropic MQA NdFeB magnet powders and a resin without any magnetic field (left) and with magnetic field (right). 


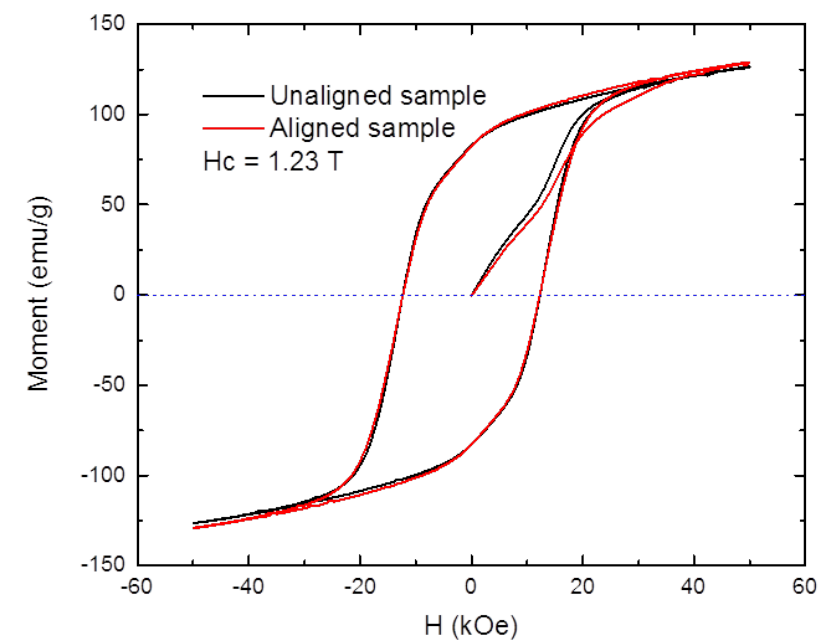

Fig. 4. Magnetic hysteresis loops of the composite magnet fabricated with anisotropic MQA NdFeB magnet powders and a resin with and without any magnetic field.

\section{Subject Inventions (As defined in the CRADA)}

The method developed for the composite magnet fabrication could be patentable. An invention disclosure based on this approach has been submitted. One manuscript based on the polymer coating of bonded magnet is published.

M. Parans Paranthaman, Christopher S. Shafer, Amy M. Elliott, Derek H. Siddel, Michael A. McGuire, Robert M. Springfield, Josh Martin, Robert Fredette, and John Ormerod, "Binder Jetting: A Novel NdFeB Bonded Magnet Fabrication,” JOM, 2016, DOI: 10.1007/s11837-0161883-4.

\section{Commercialization Possibilities}

Additively printed composite magnets offer a better option for making low cost complex shapes from isotropic and anisotropic magnet powders. Here we demonstrated a novel approach - Big Area Additive Manufacturing (BAAM) - to fabricate magnetic braces with isotropic magnet nylon composites. Further improvements are necessary with BAAM and also composite fabrication methods using anisotropic magnet samples.

\section{Plans for Future Collaboration}

Additive manufacturing offers significant advantages such as cost effectiveness (no tooling required), fast speed, and capability of producing parts of unlimited in sizes and shapes. Therefore, BAAM provides an effective method in realizing arbitrary shape with minimum cost and waste, and has the potential to revolutionize large-scale industry production of composite magnets. In the future work, the effect of binder type, loading fraction of the magnetic powder, anisotropic particles, and processing temperature on the magnetic and mechanical properties of the printed bonded magnets will be investigated. Also, large 
area composite magnet fabrication using anisotropic magnet samples will be focused. Electromagnets will be used to magnetize these composite magnets.

\section{Conclusions}

In this work, we report the feasibility of using BAAM to print magnets with complex shapes and sizes. Preliminary results on the composite magnet fabrication with anisotropic MQA NdFeB magnet powders are promising. Further process optimization is necessary to improve the magnetic properties.

\section{References}

1. M. Parans Paranthaman, Christopher S. Shafer, Amy M. Elliott, Derek H. Siddel, Michael A. McGuire, Robert M. Springfield, Josh Martin, Robert Fredette, and John Ormerod, "Binder Jetting: A Novel NdFeB Bonded Magnet Fabrication,” JOM, 2016, DOI: 10.1007/s11837-0161883-4. 